\title{
Elements and Overall Optimization of University Self-Organizing Physical Education Teaching System Based on Holistic Theory
}

\author{
Min Li and Jianwei Zhong \\ College of Physical Education, Jiangxi Normal University, Nanchang 330022, China \\ Correspondence should be addressed to Jianwei Zhong; zhongjw04@126.com
}

Received 21 December 2021; Revised 5 January 2022; Accepted 6 January 2022; Published 3 February 2022

Academic Editor: Tongguang $\mathrm{Ni}$

Copyright ( $) 2022 \mathrm{Min} \mathrm{Li} \mathrm{and} \mathrm{Jianwei} \mathrm{Zhong.} \mathrm{This} \mathrm{is} \mathrm{an} \mathrm{open} \mathrm{access} \mathrm{article} \mathrm{distributed} \mathrm{under} \mathrm{the} \mathrm{Creative} \mathrm{Commons} \mathrm{Attribution}$ License, which permits unrestricted use, distribution, and reproduction in any medium, provided the original work is properly cited.

\begin{abstract}
The fact that self-organized physical education teaching systems in universities should have diversity and imbalance has been weakened, and we need to focus on the self-thinking, self-learning, and uncertain factors in the teaching process. The components of the entire teaching system are known as teaching elements. We cannot understand the integrity of teaching elements without understanding system theory. The teaching in physical education classes is the same way. This paper analyzes the elements and overall optimization of university self-organizing physical education teaching system using the methods of literature review and logical argumentation, starting from the perspective of holism and combining with the characteristics of physical education. The effectiveness and superiority of this model are tested by comparing sports performance data with ACOA (Ant Colony Optimization Algorithm) to optimize the sports video of SVM (Support Vector Machine). The findings show that the model can improve sports performance prediction accuracy, and the prediction results are more reliable, providing useful information for sports training.
\end{abstract}

\section{Introduction}

Many experts and scholars have conducted research on college physical education teaching models in recent years and have produced some impressive results, such as inductive analysis of typical college physical education teaching models, current state of college physical education teaching models, and experimental research on typical college physical education teaching models $[1,2]$. Teaching in the classroom is a complex and comprehensive system with many components. The efficiency and quality of classroom teaching will be greatly improved if the individual vitality of these elements can be stimulated and their potential functions are fully realized [3]. Some philosophy of technology, which is emerging in the field of natural science, provides a new perspective for us to reexamine education, from the study of universal educational law to the search for situational educational significance.

There are five main elements within the university curriculum, disciplines, majors, curriculum contents, teachers, and students, which together constitute the university curriculum system [4]. However, the physical education in schools is responsible for the important physical exercise in the student period, but, through the investigation, research has found that there are many problems in college sports $[5,6]$. In recent years, some scholars have applied the perspective of holism to the scientific research of physical education, which involves the exploration of the purpose of self-organized physical education, the prospect of the transformation of physical education paradigm, and so forth, and have made a useful discussion on applying the perspective of holism to teaching and training practice [7]. However, most of the results define self-organizing systems as individual students, and there are few results in analyzing the teaching process in which teachers and students participate together as a system from the perspective of holism. From the point of view of system theory, these factors that make up physical education teaching are a whole structural system, which not only belongs to the big system of school physical education but also contains its own subsystems such 
as goals, feedback, teaching contents, and teaching methods [8]. Teaching material (teaching content system) is the carrier of teaching theory and the object of teaching practice, which plays a key role in the overall framework of physical education teaching mode.

Teaching mode is defined as "a strategic system of relatively stable teaching procedures and methods that must be followed in the teaching process, which is formed based on teaching ideas and teaching rules, and includes the combination of various elements in the teaching process, teaching procedures, and corresponding strategies" $[9,10]$. This paper builds on the nonlinear interaction between systems and the overall synergy effect to continuously develop in the direction of structure, organization, and multiple functions, from disorder to order and from order to higher order, in order to form positive influencing factors and evolutionary power and promote the optimization of physical education classroom teaching process.

\section{Related Work}

At first, the holistic perspective does not serve pedagogy subordinate to social sciences, and of course it also includes physical education, which is actually produced in natural sciences [11]. With the development of the times, more and more theories have explained and extended the holistic perspective, gradually improving the system into a group theory including many subtheories such as dissipative structure theory and synergetics, accelerating the evolution of holistic perspective in the field of natural science and laying a solid foundation for the field of auxiliary education in the future. Literature [12] holds that when interest factors occupy students' psychological tendency, students will show their physical strength and attention beyond usual and enjoy sports activities. Literature [13] holds that sports are an indispensable part in today's social activities and personal life. The sooner you realize this, the sooner you can shape a healthier body and mind. Literature [14] holds that the holistic perspective supported by many subtheories is in line with the current teaching needs. Literature [15] points out that today's traditional physical education relies too much on other organizations' interference, which limits the development of independent participation, self-thinking, and teaching methods in physical education. Literature [16] holds that it is necessary to improve the teaching methods of traditional teaching, which should be the center of our attention, highlight the subjectivity of students, and better impart knowledge. Literature [17] points out that although these traditional physical education teaching modes have played a certain role in the development of school physical education in China, they also show disadvantages in teaching; that is, they pay too much attention to sports technology learning and neglect the development of students' interests and the cultivation of independent thinking ability, which leads to some single physical education teaching methods. In literature $[18,19]$, by comparing the current teaching model with the needs of post-moderncurriculum reform, it is concluded that, nowadays, the traditional teaching mode has certain limitations, focusing on teaching, simplifying the teaching mode, and neglecting the cultivation of students' other qualities. However, modern physical education develops the teaching function of physical education from various angles, focusing on learning, implementing democratic teaching, and no longer sticking to a teaching mode but paying more attention to giving full play to students' learning initiative.

There are numerous sports video classifications available today. People used expert systems to classify sports videos at first. Expert systems, on the other hand, have high knowledge requirements and are unable to solve new sports video classification problems. At the moment, they are mostly computer-based automatic sports video classification methods [20]. According to literature [21], the current video similarity detection system has the problem of detection speed not being able to keep up with the actual production demand. The authors built a distributed platform for batch video similarity detection using cloud computing and the compound eye of insects, and the detection speed was greatly improved. Literature [22] created a distribution-based video content analysis platform. Offline and real-time video analyses are both possible with the platform. The platform is used in the testing stage to detect shot boundaries of multiple video segments at the same time, with a detection efficiency that is higher than that of a single machine. Literature [23] designed and implemented a distributed video processing platform that combines Storm and Hadoop, two distributed computing frameworks. In [24], a single frame image of video is parallel processed using Hadoop, and each frame block of the image is parallel processed using GPU. Literature [25] proposed a real-time video processing cloud platform based on embedded devices, on which Storm was deployed as a cloud computing environment and video streams were processed using the DL (Deep Learning) algorithm. Literature [26] proposed a video processing framework based on two cloud computing technologies, Storm streaming and Hadoop batch processing, and deep learning was employed to extract high-level semantic features from video data.

\section{Research Method}

3.1. Analysis of Various Elements of Physical Education. People usually equate the system with the whole. In fact, the system is a description of the whole. What people really care about is not the system but the whole. The purpose and essence of people focusing on the system lie in the whole. The essence of systematic holistic thinking is its wholeness, and system is actually an expression of wholeness; that is to say, from a systematic point of view, the wholeness of everything can be expressed by systematic terms and means. This is the system holism that we want to discuss.

From the static structure point of view, teaching is simply the activity where teachers teach students teaching content. Without any of these three, it cannot be called teaching. Without students, there is no teaching object; without teachers, teaching will lose its dominance; without teaching content, teachers and students will lose their teaching support. Trend shows that, to respect and 
develop students' personality, and attach importance to students' dominant position, insistence on diversification, flexibility, and individualization of teaching organization forms is a must. Therefore, letting students choose to participate in teaching classes with different project combinations in different periods can fully mobilize their learning enthusiasm, help to stimulate their interest and enthusiasm in learning physical education, and effectively promote the realization of the best teaching scheme.

In physical education teaching, good ideological quality is classified as the level of basic physical education quality. First, good ideological quality helps students to establish a correct world outlook, values, and outlook on life and plays a benchmark role for students to form a correct view of physical education quality. Second, with the rapid development of computer technology and information technology, various ideas and opinions are constantly emerging, students are in the whirlpool of information, and good ideological quality can improve students' cognitive ability. Of course, attention should also be paid to the teacher's self-development. In addition, the close cooperation between teachers can give students a better influence. Physical education teachers should investigate from many aspects such as skill level and sports theory. In teaching practice, they should not only teach students professional technical movements and sports theory knowledge but also cultivate students' good moral and will qualities.

A self-organizing physical education teaching system is an organic whole with specific functions which is made up of several interconnected and interacting elements. People are the main body of physical education and the main factor of the teaching system and the driving force behind the system's self-organization development. Individual differences as well as an overall identity exist among the various elements. Individual differences manifest as competition, whereas overall identity takes the form of synergy. They rely on one another, interact with and complement one another, and, through the teaching process, produce the overall effect (see Figure 1).

The model shown in Figure 1 shows that the integrity of the self-organizing system is the basic condition for this system to keep its own unique stipulation in motion. As a system, college physical education is a multilevel whole, which is not the simple addition of all the components but the organic combination of all the components. It reveals the distinctive characteristics where the interaction between teachers and students dominates the overall evolution of the teaching system. The main element of the physical education system is people, which has the characteristics of "initiative" and "liveliness." It is the key element of the system and plays a leading role in the operation and development of the system.

The course content of university is a process of getting rid of the rough and getting the fine in the selection of knowledge, and it does not form a direct correspondence with human activities. In addition, the course content is internalized into students' own knowledge structure through students' own choice and reconstruction in the process of transmission, and it is impossible to fully meet the university curriculum objectives. The integrated power thus formed is the leading force to promote the overall evolution and development of the teaching system. This is a new understanding of the integrity of the teaching system beyond the traditional holistic theory, and it is the essence of the integrity characteristics of the self-organized physical education teaching system.

The evolution and change of discipline is the most important factor in the evolution and change of the five elements of curriculum, and its fluctuation will inevitably lead to a change in specialty setting, followed by the selection and reorganization of learning materials, and a change in the numbers of teachers and students. The external environment of universities, particularly the development and changes of scientific knowledge, influences the development and changes of disciplines. Human cognition of the world produces scientific knowledge. It has a level and order of evolution, and it is evolving from a simple low level to a complex high level. As a result, it establishes the timeline for the evolution and change of the university curriculum system.

\subsection{Overall Optimization of the University Self-Organizing} Physical Education Teaching System. The system goes through ups and downs, producing random branches at the critical point, so as to achieve a new balance and order among the elements of the curriculum structure. This is the key to whether the university curriculum can realize its function. Relatively stable curriculum structure is an inevitable process of curriculum self-organization. The orderliness of university curriculum structure is manifested in "spatial orderliness" and "temporal orderliness." Therefore, the self-organization control of school physical education teaching must take into account the cultivation and combination of students' emotions, attitudes, and interests from the overall perspective of the system, so that students can apply what they have learned about physical education to practice.

With the advancement of multimedia technology, there are more and more types of image acquisition devices available, as well as an increase in the number of people participating in sports. Every day, mobile phones and cameras can collect a large number of sports videos, posing challenges for sports video management $[1,2]$. The following is the principle of ACOA (Ant Colony Optimization Algorithm) optimized SVM (Support Vector Machine) sports video classification: To begin, sports videos are gathered, and a variety of sports video classification features are extracted. The features of sports video classification are then processed using the principal component analysis algorithm. A sports video classification model is established using SVM as the input and sports video category as the output, and the SVM is optimized by ACOA to produce the best sports video classifier.

SVM maps sports video classification samples by function $\phi(x)$ and then carries out the following processing in high-dimensional space: 


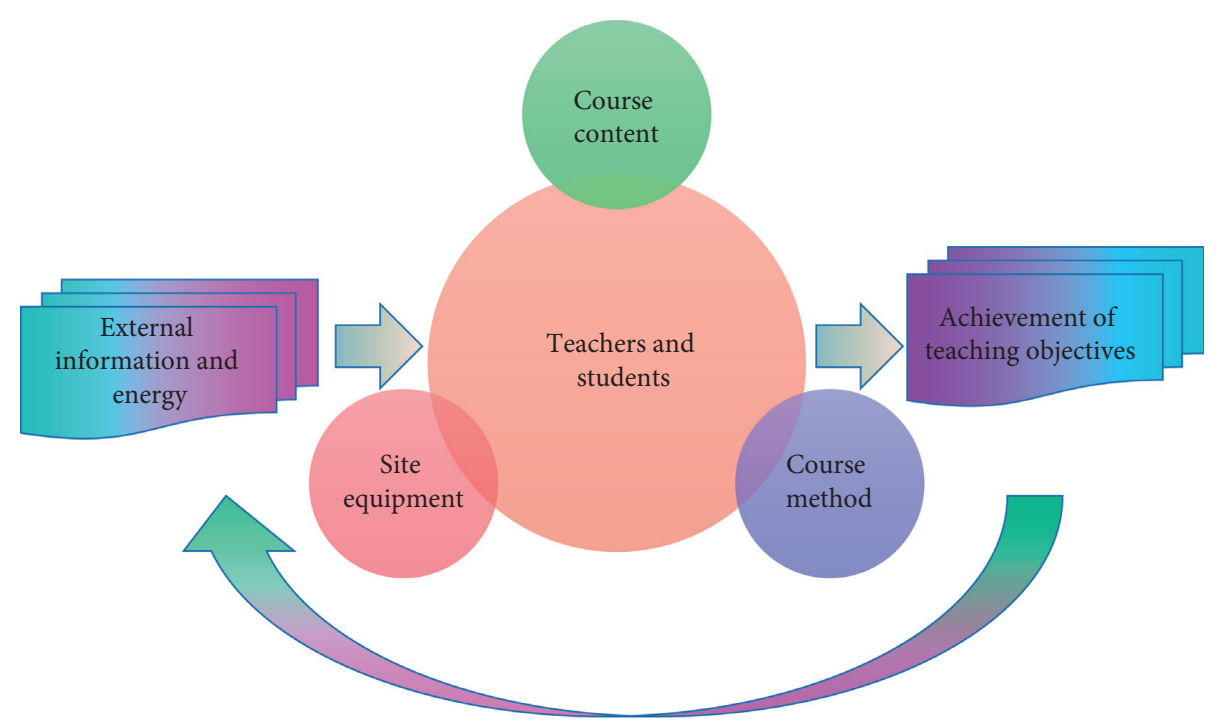

FIgURE 1: Sustainable operation of the university self-organizing physical education teaching system.

$$
\begin{aligned}
& \min _{\omega, b, \xi} J\left(\omega, \xi_{i}\right)=\frac{1}{2} \omega^{T} \cdot \omega+C \sum_{i=1}^{l} \xi_{i}^{2}, \\
& \text { s.t. }\left\{\begin{array}{l}
y_{i}=\omega^{T} \cdot \omega\left(x_{i}\right)+b+\xi_{i}, \\
\xi_{i} \geq 0, \\
i=1,2, \ldots, l,
\end{array}\right.
\end{aligned}
$$

where $\xi_{i}^{2}$ is the classification error and $C$ is the penalty parameter.

According to the pheromone size left by ants, the next transfer probability of each ant is determined as follows:

$$
p(i)=\frac{e^{T_{0}(\text { BestIndex })-T_{0}(i)}}{e^{T_{0}(\text { BestIndex })}},
$$

where BestIndex represents the maximum pheromone concentration.

The pheromone depth is updated by the following formula:

$$
T_{0}(i)=(1-\rho) * T_{0}(i)+\Delta(i) .
$$

Task scheduling is the process of allocating the best resources for the tasks that need to be completed, taking into account various factors such as time, cost, and resource utilization, in order to reduce task completion time and improve system resource utilization. HPSO (Hybrid Particle Swarm Optimization) is used to schedule the platform's tasks, with the goal of reducing task completion time as the algorithm's optimization goal. Figure 2 illustrates an HPSObased task scheduling model.

For users, the main process of using the platform to process tasks is as follows:

(1) Initiate a task processing request to the user platform
(2) After receiving the user's task processing request, the task management module adds the task to the task queue

(3) The task scheduling module uses HPSO calculation to obtain the best task allocation scheme based on the status information of the computing cluster and the information of tasks to be processed

(4) The task distribution module distributes tasks to specific computing nodes for processing according to the scheduling scheme generated by the task scheduling module

(5) After the task processing is finished, the node feeds back the calculation result to the task management module, and the task management module informs the user that the task processing is finished

Assuming that there are $N$ tasks and $M$ virtual machines, the length of the particle position information is $N$, and the value of each element of the particle position is within the range of $[1, M]$. Formula (4) is an example of particle position coding.

$$
X=\left\{x_{1}, x_{2}, \ldots, x_{j}, \ldots, x_{N}\right\}, \quad 1 \leq x_{i} \leq M,
$$

where $X$ represents the position information of a particle in the population, and for it contains any one-digit value $x_{j}, j$ represents the task number, and $x_{i}$ represents the virtual machine assigned to the task number $i$.

As all virtual machines process in parallel, the completion time of the task is the longest time needed to run among all virtual machines, and the calculation method is shown in the following formula:

$$
\text { completeTime }=\max \left\{\text { totalTime }_{i}\right\}, \quad i=1,2, \ldots, M \text {. }
$$

Because the algorithm takes reducing the task completion time as the optimization goal, its corresponding fitness 


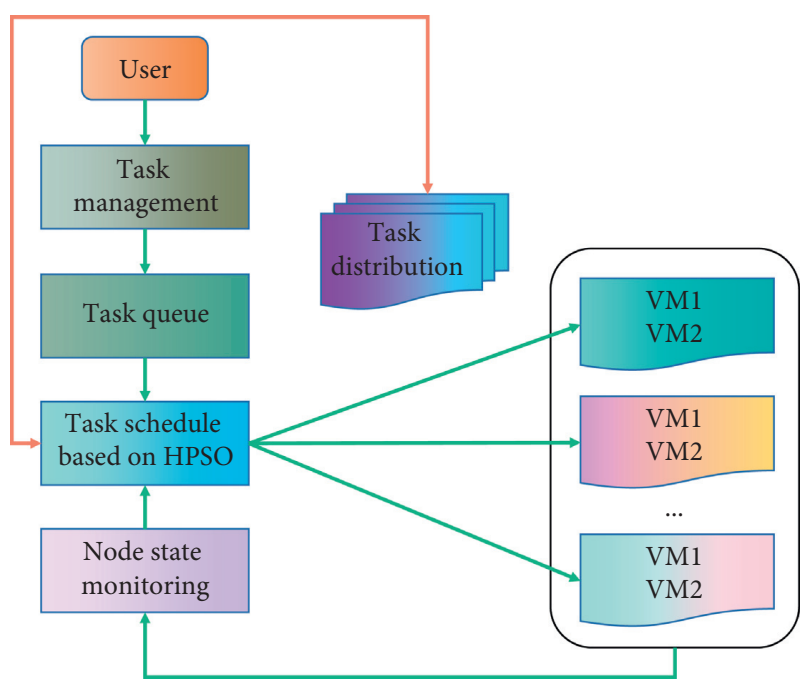

Figure 2: Task scheduling model based on HPSO.

function is shown in formula (6). The less the task completion time, the larger the fitness value.

$$
\text { Fit }=\frac{1}{\text { completion time }} \text {. }
$$

According to the number of iterations, the inertia weight is dynamically updated, and the velocity and position of each particle are simultaneously updated, and the best historical position of each particle and the global best position of the population are recorded.

Collect the sports performance data and process it with the following formula:

$$
\hat{x}_{i}=\frac{\left(x_{i}-x_{\min }\right)}{\left(x_{\max }-x_{\min }\right)},
$$

where $\hat{x}_{i}$ is the sports result after processing; $x_{i}$ is the original sports achievement; and $x_{\max }$ and $x_{\min }$ represent the maximum and minimum values, respectively.

After the prediction, the prediction results of sports performance are processed by the following formula:

$$
x_{i}=\hat{x}_{i} \times\left(x_{\max }-x_{\min }\right)+x_{\min } .
$$

According to the fitness function, the positions of fireflies are evaluated. Fireflies with weak attraction move to those with strong attraction, and the positions of all fireflies are updated.

\section{Results Analysis and Discussion}

The acquisition of high-level knowledge in the learning process is dependent not only on teachers' instruction but also on students' ability to learn on their own. Others organize the former, while learners organize themselves for the latter. Self-organization does not rely solely on students but rather requires the guidance and intervention of teachers. In the field of social sciences, self-organization demonstrates its scientificity, emphasizing the importance of guiding students to establish an orderly knowledge system, improving students' interest in learning, and promoting students' development as the fundamental idea of teaching design. The pretest and posttest experiments of EG (experimental group) are compared after the teaching experiment. The comparison results of the EG pretest and posttest experiments are shown in Figure 3.

According to the self-comparison results in Figure 3, it is shown that the open and cooperative teaching method from the perspective of holism can effectively improve students' learning interest, autonomous participation in class, and autonomous learning after class. After years of studying and living on campus, students have long been full of rejection of the teaching environment organized by him, so students regard physical education class as an opportunity to liberate themselves. When students find that sports still need a lot of repeated learning, students will naturally have resistance.

In order to maintain this achievement, it is necessary to enrich the teaching constantly, so that physical education can have more choices in content selection and purpose setting. From passive learning to active learning, we get the freedom and autonomy we deserve. On the basis of developing sports technology and maintaining physical and mental health, the openness of physical education is to develop students' subjectivity by using a more open teaching environment and a coordinated teaching atmosphere, so that the disorderly knowledge system can build an orderly knowledge structure in the open environment.

After the statistics, CG (control group) compares its own pretest and posttest experiments. Figure 4 shows the comparison results of CG pretest and posttest experiments.

After seeing the experiment, the scores of CG in four dimensions have slightly improved, indicating that, regardless of the teaching method used in physical education, students' interest in physical education learning can be significantly improved, but self-organized teaching can greatly improve the teaching effect in a short period of time. Five types of sports video data were chosen as the experimental objects in order to test the ACOA optimized SVM's sports video classification effect. Learn the training samples, establish corresponding sports video classifiers, and then classify the verification samples and count their sports video classification accuracy using the ACOA optimized SVM, the sports video classification method without parameter optimized SVM, and the sports video classification method of BPNN (BP neural network). The results are shown in Figure 5.

It can be seen from Figure 5 that this method can effectively reduce the error rate of sports video classification and obtain better sports video classification results.

The development of students' sports knowledge and skills and the construction of physical and mental health are all influenced by positive psychological tendencies. The formation and cultivation of autonomy are not only the purpose of teaching but also an indispensable feature for every member of society to move towards the continuous development of society. The inherent law of cultivation and students' self-organizing ability in the process of physical education study and exercise allow physical education to better cooperate with quality education and effectively develop students' physical and mental qualities. 


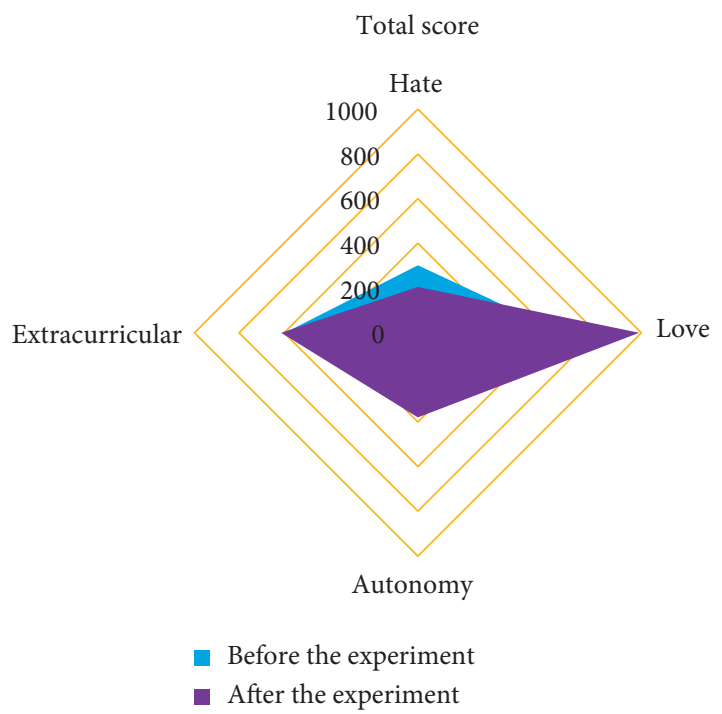

Figure 3: Comparison results of EG pretest and posttest experiments.

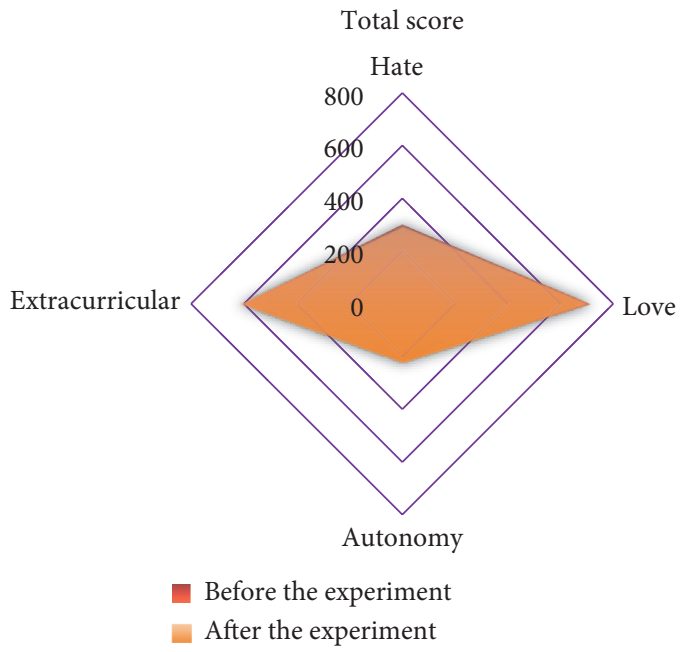

FIGURE 4: Self-comparison chart of CG pretest and posttest experiments.

Statistics of sports video classification time of the three methods are shown in Figure 6.

As can be seen from Figure 6, this method reduces the time of sports video classification and accelerates the speed of sports video classification.

Open physical education teaching demonstrates a wide range of learning objectives, all of which are complex and challenging. Students are part of a fast-paced information delivery system that is rich in differences and innovations. Knowledge is constantly being refreshed, new action structures are constantly being generated, and there is always something new to discover. In this way, knowledge gradually develops from disorder to order in a teaching situation with independent thinking as the primary component, and a reasonable external intervention force will aid in the formation of orderly knowledge. In the collective, there will be collaboration and competition. This migration force will be infinitely magnified and deeply portrayed in the minds of other students once an individual's technology model is recognized by the majority of people in the collective. Students who differ from the recognized technology model will consciously imitate and learn or even want to outperform their competitive behavior, develop autonomous and spontaneous learning, and then form an orderly and effective knowledge structure from a disorderly structure.

In order to verify the superiority of HPSO algorithm in convergence speed, it is compared with the algorithms in literature [22], literature [22], and literature [24]. A comparative experiment was conducted with 20 virtual computing resources and 500 tasks. The experimental results are shown in Figure 7.

When the number of iterations is 20 , the task completion time of HPSO is obviously less than that of [22]. The specific reason is that the random method is used to initialize particle swarm in the algorithm of [22]. However, HPSO uses the method based on chaos opposition to initialize the population, which improves the diversity of the population, and makes the algorithm have a greater chance of searching for the relative optimal solution in the early stage than in the literature [22].

In the experiment, the number of tasks remained unchanged at 600 , and the number of virtual machines was 10 , $20,30,40$, and 50 , respectively. The task completion times required by literature [22], literature [24], and HPSO were compared. All the experiments were repeated five times, and the average completion time of five times was taken as the experimental result, as shown in Figure 8.

Literature [24] introduced the mutation operation of genetic algorithm into PSO, which ensured the diversity of population in the iterative process. HPSO also introduces the centroid of particle swarm to keep the diversity of the population. In addition, it uses chaos-based and oppositionbased learning to initialize the population, which makes the initial diversity of the population higher, thus making the algorithm more likely to obtain the optimal solution.

Students are allowed to play freely for the sake of a broad teaching range. Teachers will guide and improve students' thinking as they take effective actions or responses to the teaching contents, in order to increase their participation, strengthen their self-learning, increase their interest in physical education, and guide them to improve their existing knowledge structure through mutual cooperation and reasonable competition in physical education. Physical development of students can be accelerated, and classroom quality can be improved. How students' states change over time is observed, breakthrough points are identified, students are encouraged to keep approaching these breakthrough points, and, finally, the conditions for technological progress are created. The teacher is allowed to lead the students through a sudden shift in their interests.

In order to improve the prediction accuracy of sports performance, a sports performance prediction model based on firefly optimized neural network is proposed to solve the problem of determining the connection weights and thresholds of BPNN. Grey model, BPNN, and RBF neural network were selected for comparative test, and $1000 \mathrm{~m}$ running result was selected as the experimental object. Their results are shown in Figure 9. 


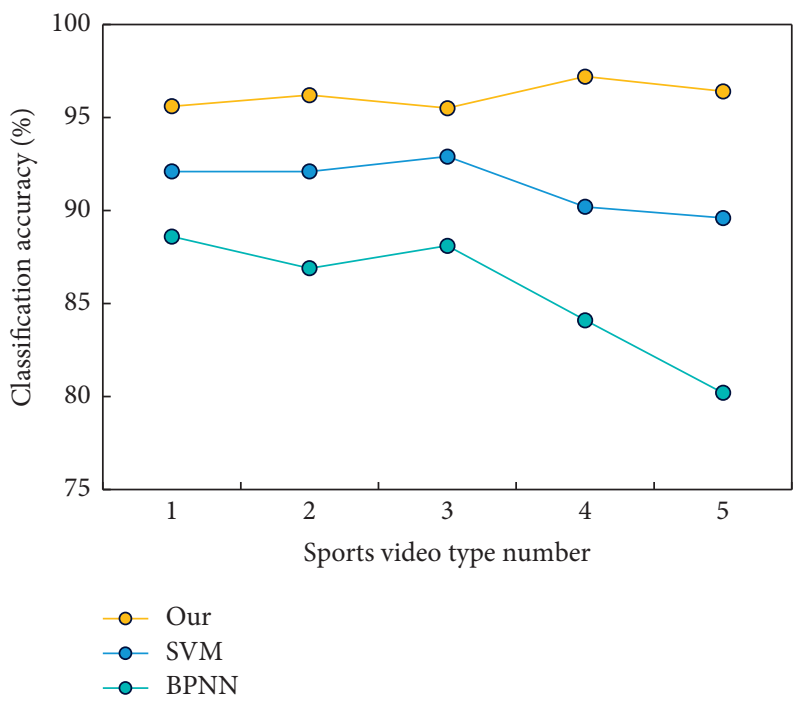

FiguRE 5: Comparison of accuracy rate of sports video classification.

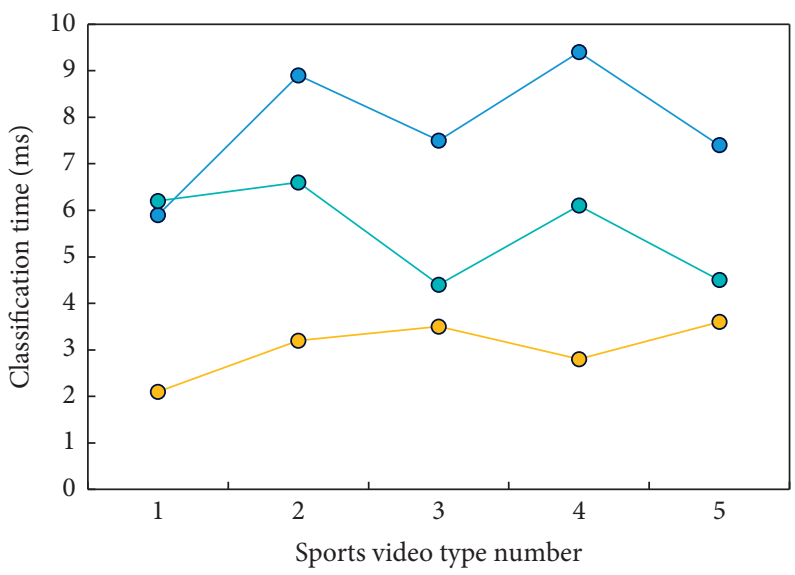

- Our

- SVM

- BPNN

FIgURE 6: Comparison of sports video classification time.

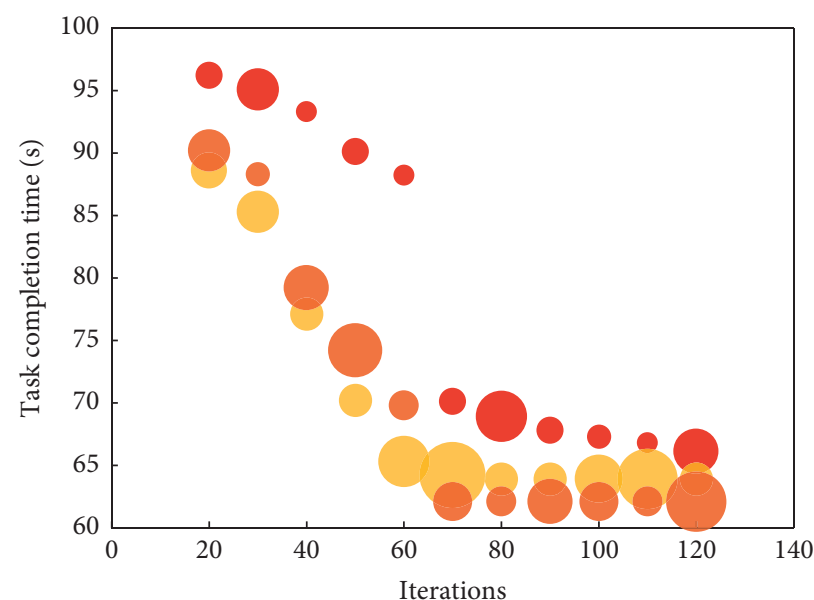

- Literature [22]

- Literature [24]

- HPSO

FIGURE 7: Comparison of convergence speed of different algorithms. 


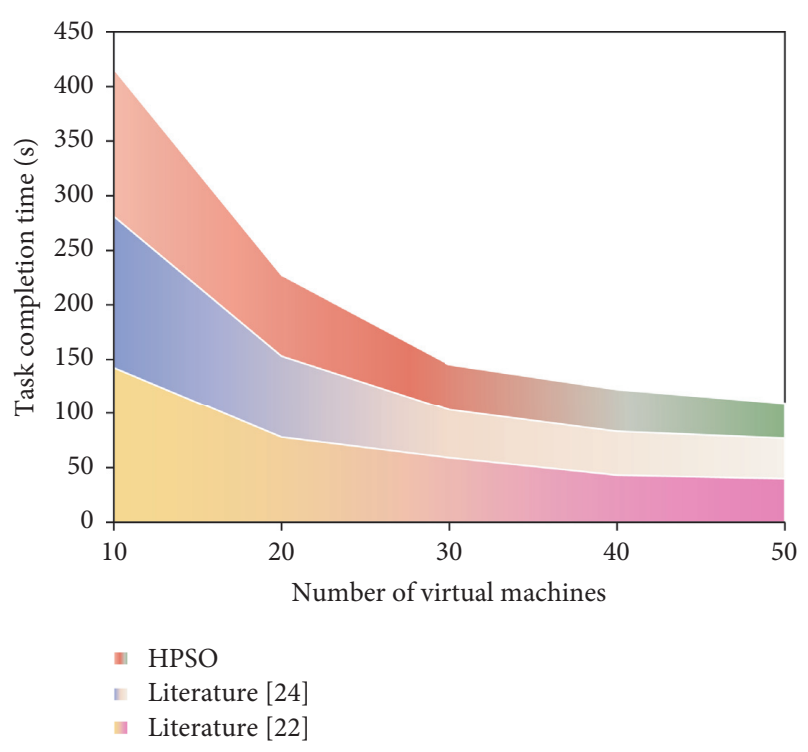

Figure 8: Comparison of task completion time under different virtual machines.

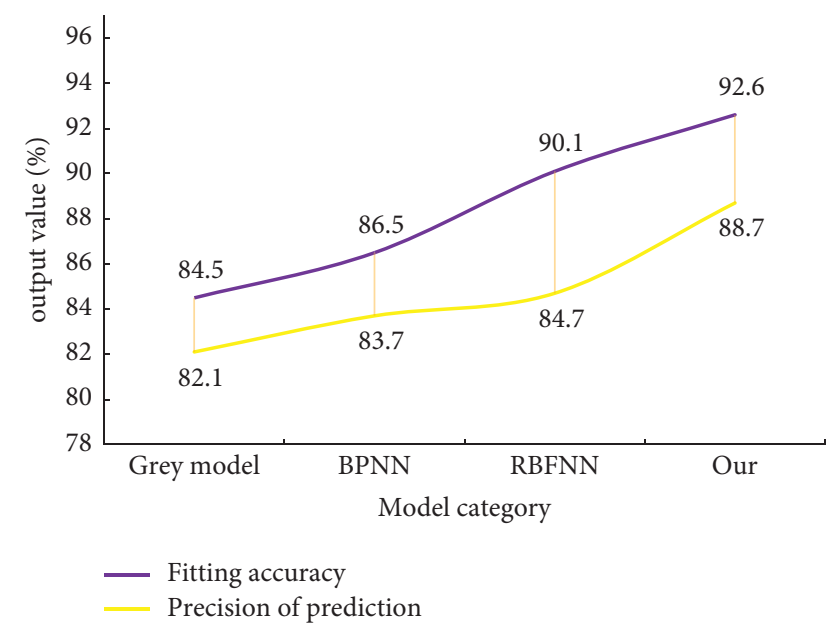

Figure 9: Universal test results.

As can be seen from Figure 9, the performance of athletes' performance fitting and prediction of this model is better than that of the comparison model.

In a physical education class, because students have to constantly adapt to the needs of teaching content, it is essential to adjust students' heart rate. Only under the appropriate heart rate condition can students' practice achieve the expected effect. In addition, because physical exercise is a gradual process in physical education, the sports load system not only reasonably grasps its rhythm in one class but also needs to consider the size of sports load according to the arrangement of sports activities (morning exercises, recess exercises, physical education class, extracurricular sports activities, amateur training, and competition activities) and teaching activities in a week to promote the healthy development of students.

Each school's situation is unique, and the physical education teaching mode is similarly unique. A comprehensive university, for example, with a large scale, multiple campuses, and a wide range of disciplines, may not be able to meet the needs of all students by using just one teaching model. Different disciplines and training goals, on the other hand, necessitate that teaching models reflect their own uniqueness on the basis of commonality. Several small groups of friends or classmates with varying levels, hobbies, and needs to practice are allowed, so that the interpersonal relationship between students and teachers is cordial and harmonious, boring and monotonous exercises become lively and interesting, and students' creative and enterprising motivation is stimulated, thus improving the teaching quality of physical education class.

\section{Conclusion}

For a long time, self-organization control theory has been widely used in the fields of nonlinear science and natural science, but there has been very little research into its application in college physical education. We should start with the teaching objectives, refine the objective structure, and create a multilevel objective system when teaching physical education classes. A new concept of physical education is formed based on the holistic theory, which attempts to establish a physical education teaching paradigm that focuses on students' subjective development and diversified transformation. The results of the HPSO algorithm show that it has some advantages over other algorithms in terms of task completion time and convergence speed. Sports video classification has a higher accuracy rate than other current sports video classification methods, and the error rate is controlled within the scope of sports video application, which overcomes the limitations of other sports performance prediction models, and the prediction results are more reliable, which can improve the scientific decisionmaking basis for sports training.

\section{Data Availability}

The data used to support the findings of this study are included within the article.

\section{Conflicts of Interest}

All the authors declare no conflicts of interest.

\section{Acknowledgments}

This study was supported by Sports Participation on the Social Integration of Urban Immigrant Groups (11CTY008).

\section{References}

[1] W. Jian, S. Bo, and X. Luo, "Validation of a teachers' achievement goal instrument for teaching physical education." Journal of Teaching in Physical Education, vol. 37, no. 1, pp. 1-27, 2017.

[2] Z. Xu, "An analysis of school physical education teaching strategies under the background of living," International Journal of Social Science and Education Research, vol. 3, no. 3, pp. 29-33, 2020. 
[3] Y. Gong, "Physical education teaching reform in colleges and universities under the concept of leisure sports," Research frontiers of education: Chinese and English versions, vol. 9, no. 3, p. 4, 2019.

[4] L. Chen and Y. Li, "The significance and practical path of outward bound training in college physical education teaching," Management Science and Research: Chinese and English Version, vol. 8, no. 1, p. 5, 2019.

[5] H.-S. Kim and Y.-K. Lee, "The relationship between recognition of physical education teaching behavior and class Attitude and school life satisfaction on middle school Student"s," Korean Journal of Sports Science, vol. 27, no. 2, pp. 789-801, 2018.

[6] H.-H. Tung, "[Embracing renovation and innovation during the pandemic: application of virtual simulation technology in nurse practitioner education]," $\mathrm{Hu} \mathrm{Li} Z \mathrm{Za} Z \mathrm{Zhi}$, vol. 68, no. 5, pp. 7-12, 2021.

[7] J. D. Wyant, "Exploring pre-service physical education teacher technology use during student teaching," Journal of Teaching in Physical Education, vol. 36, no. 2, pp. 173-184, 2017.

[8] D. Ping and A. Li, "Simulation of physical education teaching based on FPGA and wearable VR equipment," Microprocessors and Microsystems, vol. 81, no. 3, Article ID 103773, 2020.

[9] C. R. Stolberg, L. H. Mundbjerg, P. Funch-Jensen, B. Gram, E.-M. Bladbjerg, and C. B. Juhl, "Effects of gastric bypass surgery followed by supervised physical training on inflammation and endothelial function: a randomized controlled trial," Atherosclerosis, vol. 273, pp. 37-44, 2018.

[10] A. Ismail, "Physical training and ocular yogic exercise in home: good alternative options to control the high-tension form of primary open angle glaucoma during the repeated COVID-19 waves," International Maritime Health, vol. 72, no. 3, pp. 243-244, 2021.

[11] K. E. Yee, J. B. Moore, G. Grieve, K. Hucks, and D. Bornstein, "Assessing value of physical training for tactical athletes," Medicine \& Science in Sports \& Exercise, vol. 52, no. 7S, p. 375, 2020.

[12] K. Koumaditis, F. Chinello, P. Mitkidis, and S. Karg, "Effectiveness of virtual vs. Physical training: the case of assembly tasks, trainer's verbal assistance and task complexity," IEEE Computer Graphics and Applications, vol. 40, no. 5, pp. 41-56, 2020.

[13] A. Lewicka, S. Lewicki, A. Kłos, B. Dębski, T. Kurył, and J. Bertrandt, "Influence of protein deficient diet, vitamin B2 supplementation and physical training on serum composition of polyunsaturated fatty acids (PUFAs) in rats," Annals of Agricultural and Environmental Medicine, vol. 24, no. 2, pp. 185-189, 2017.

[14] S. Suárez-García, J. M. Del Bas, A. Caimari, R. M. Escorihuela, L. Arola, and M. Suárez, "Impact of a cafeteria diet and daily physical training on the rat serum metabolome," Plos One, vol. 12, no. 2, Article ID e0171970, 2017.

[15] M. Smeda, K. Przyborowski, B. Proniewski et al., "Breast cancer pulmonary metastasis is increased in mice undertaking spontaneous physical training in the running wheel; A call for revising beneficial effects of exercise on cancer progression," American Journal of Cancer Research, vol. 7, no. 9, pp. 1926-1936, 2017.

[16] K. D. Maida, A. C. Gastaldi, T. de Paula Facioli, J. E. de Araújo, and H. C. D. de Souza, "Physical training associated with Enalapril but not to Losartan, results in better cardiovascular autonomic effects," Autonomic Neuroscience, vol. 203, pp. 33-40, 2017.
[17] C. M. Faleiros, H. D. C. Francescato, M. Papoti et al., "Effects of previous physical training on adriamycin nephropathy and its relationship with endothelial lesions and angiogenesis in the renal cortex," Life Sciences, vol. 169, pp. 43-51, 2017.

[18] T. L. Grier, M. Canham-Chervak, M. K. Anderson, T. T. Bushman, and B. H. Jones, "Effects of physical training and fitness on running injuries in physically active young men," The Journal of Strength \& Conditioning Research, vol. 31, no. 1, pp. 207-216, 2017.

[19] H. Arrieta, C. Rezola-Pardo, S. M. Gil, J. Irazusta, and A. Rodriguez-Larrad, "Physical training maintains or improves gait ability in long-term nursing home residents: a systematic review of randomized controlled trials," Maturitas, vol. 109, pp. 45-52, 2018.

[20] F. C. M. Melo, K. K. F. de Lima, A. P. K. F. Silveira et al., "Physical training and upper limb strength of people with paraplegia: a systematic review," Journal of Sport Rehabilitation, vol. 1, pp. 1-20, 2018.

[21] S. Ling, Z. Cai, L. Li, and K. Lu, "Performance evaluation of deep feature learning for RGB-D image/video classification," Information Sciences, vol. 385-386, no. C, pp. 266-283, 2017.

[22] A. Chadha, A. Abbas, and Y. Andreopoulos, "Video classification with CNNs: using the codec as a spatio-temporal activity sensor," IEEE Transactions on Circuits and Systems for Video Technology, vol. 7, no. 99, 2017.

[23] V. Mygdalis, A. Iosifidis, A. Tefas, and I. Pitasa, "Semi-Supervised subclass support vector data description for image and video classification," Neurocomputing, vol. 278, pp. 51-61, 2017.

[24] S. Zebhi, S. Al-Modarresi, and V. Abootalebi, "Converting video classification problem to image classification with global descriptors and pre-trained network," IET Computer Vision, vol. 14, no. 8, pp. 614-624, 2020.

[25] J. Gao and C. Xu, "CI-GNN: building a category-instance graph for zero-shot video classification," IEEE Transactions on Multimedia, vol. 22, no. 12, pp. 3088-3100, 2020.

[26] L. Zhu and Y. Yang, "Label independent memory for semisupervised few-shot video classification," IEEE Transactions on Pattern Analysis and Machine Intelligence, vol. 44, no. 1, pp. 273-285, 2020. 